\title{
Existence of Positive Solution for BVP of Nonlinear Fractional Differential Equation
}

\author{
Jun-Rui Yue, ${ }^{1}$ Jian-Ping Sun, ${ }^{1}$ and Shuqin Zhang ${ }^{2}$ \\ ${ }^{1}$ Department of Applied Mathematics, Lanzhou University of Technology, Lanzhou 730050, China \\ ${ }^{2}$ Department of Mathematics, China University of Mining and Technology, Beijing 100083, China \\ Correspondence should be addressed to Jian-Ping Sun; jpsun@lut.cn
}

Received 16 July 2015; Accepted 14 September 2015

Academic Editor: Chris Goodrich

Copyright (c) 2015 Jun-Rui Yue et al. This is an open access article distributed under the Creative Commons Attribution License, which permits unrestricted use, distribution, and reproduction in any medium, provided the original work is properly cited.

We consider the following boundary value problem of nonlinear fractional differential equation: $\left({ }^{C} D_{0+}^{\alpha} u\right)(t)=f(t, u(t)), t \in$ $[0,1], u(0)=0, u^{\prime}(0)+u^{\prime \prime}(0)=0, u^{\prime}(1)+u^{\prime \prime}(1)=0$, where $\alpha \in(2,3]$ is a real number, ${ }^{C} D_{0+}^{\alpha}$ denotes the standard Caputo fractional derivative, and $f:[0,1] \times[0,+\infty) \rightarrow[0,+\infty)$ is continuous. By using the well-known Guo-Krasnoselskii fixed point theorem, we obtain the existence of at least one positive solution for the above problem.

\section{Introduction}

Fractional calculus has demonstrated applications in numerous seemingly diverse and widespread fields of science and engineering, for example, fluid flow, rheology, electrical networks, chemical physics, control theory of dynamical systems, optics, and signal processing [1].

Since the discussion of many problems can be summed up in the study of boundary value problems (BVPs for short) for nonlinear fractional differential equations, recently, the existence or uniqueness of solutions or positive solutions of BVPs for nonlinear fractional differential equations has received much attention from many authors; see [2-13] and the references therein.

In particular, Zhang [6] studied the existence and multiplicity of positive solutions to the following BVP of nonlinear fractional differential equation:

$$
\begin{aligned}
& \left({ }^{C} D_{0+}^{\alpha} u\right)(t)=f(t, u(t)), \quad t \in(0,1), \\
& u(0)+u^{\prime}(0)=0, \\
& u(1)+u^{\prime}(1)=0,
\end{aligned}
$$

where $\alpha \in(1,2]$ is a real number and ${ }^{C} D_{0+}^{\alpha}$ denotes the standard Caputo fractional derivative. The main tools used were the Guo-Krasnoselskii and Leggett-Williams fixed point theorems.
In 2009, by using the nonlinear alternative of LeraySchauder type and Guo-Krasnoselskii fixed point theorem, Bai and Qiu [12] discussed the existence of a positive solution to the following singular BVP of nonlinear fractional differential equation:

$$
\begin{aligned}
& \left({ }^{C} D_{0+}^{\alpha} u\right)(t)+f(t, u(t))=0, \quad t \in(0,1), \\
& u(0)=u^{\prime}(1)=u^{\prime \prime}(0)=0,
\end{aligned}
$$

where $\alpha \in(2,3]$ is a real number, ${ }^{C} D_{0+}^{\alpha}$ denotes the standard Caputo fractional derivative, and $f(t, u)$ is singular at $t=0$.

Motivated greatly by the above-mentioned works, in this paper, we consider the following BVP of nonlinear fractional differential equation:

$$
\begin{aligned}
\left({ }^{C} D_{0+}^{\alpha} u\right)(t) & =f(t, u(t)), \quad t \in[0,1], \\
u(0) & =0 \\
u^{\prime}(0)+u^{\prime \prime}(0) & =0 \\
u^{\prime}(1)+u^{\prime \prime}(1) & =0
\end{aligned}
$$

where $\alpha \in(2,3]$ is a real number, ${ }^{C} D_{0+}^{\alpha}$ denotes the standard Caputo fractional derivative, and $f:[0,1] \times[0,+\infty) \rightarrow$ $[0,+\infty)$ is continuous. First, Green's function $G(t, s)$ for the associated linear BVP is constructed. It is necessary to point 
out that $G(t, s)$ is singular at $s=1$. Next, some useful properties of $G(t, s)$ are studied. Finally, existence results of at least one positive solution for BVP (3) are obtained.

In order to obtain our main results, we need the following Guo-Krasnoselskii fixed point theorem [14, 15].

Theorem 1. Let $E$ be a Banach space and let $K$ be a cone in $E$. Assume that $\Omega_{1}$ and $\Omega_{2}$ are bounded open subsets of $E$ such that $0 \in \Omega_{1}, \bar{\Omega}_{1} \subset \Omega_{2}$, and let $T: K \cap\left(\bar{\Omega}_{2} \backslash \Omega_{1}\right) \rightarrow K$ be a completely continuous operator such that either

(1) $\|T u\| \leq\|u\|$ for $u \in K \cap \partial \Omega_{1}$ and $\|T u\| \geq\|u\|$ for $u \in K \cap \partial \Omega_{2}$ or

(2) $\|T u\| \geq\|u\|$ for $u \in K \cap \partial \Omega_{1}$ and $\|T u\| \leq\|u\|$ for $u \in K \cap \partial \Omega_{2}$.

Then $T$ has a fixed point in $K \cap\left(\bar{\Omega}_{2} \backslash \Omega_{1}\right)$.

\section{Preliminaries}

For the convenience of the reader, we present here some necessary definitions and results from fractional calculus theory. These definitions and results can be found in the recent literature [1].

In this section, we always assume that $\mathbb{N}=$ $\{1,2,3, \ldots\}, \alpha>0$ and $[\alpha]$ denotes the integer part of $\alpha$.

Definition 2. The Riemann-Liouville fractional integral $I_{0+}^{\alpha} u$ of order $\alpha$ is defined by

$$
\left(I_{0+}^{\alpha} u\right)(t):=\frac{1}{\Gamma(\alpha)} \int_{0}^{t} \frac{u(s) d s}{(t-s)^{1-\alpha}}, \quad t>0 .
$$

Definition 3. The Riemann-Liouville fractional derivative $D_{0+}^{\alpha} u$ of order $\alpha$ is defined by

$$
\begin{aligned}
\left(D_{0+}^{\alpha} u\right)(t) & :=\frac{d^{n}}{d t^{n}}\left(I_{0+}^{n-\alpha} u\right)(t) \\
= & \frac{1}{\Gamma(n-\alpha)} \frac{d^{n}}{d t^{n}} \int_{0}^{t} \frac{u(s) d s}{(t-s)^{\alpha-n+1}}, \\
& n=[\alpha]+1, t>0 .
\end{aligned}
$$

Definition 4. The Caputo fractional derivative ${ }^{C} D_{0+}^{\alpha} u$ of order $\alpha$ is defined via the above Riemann-Liouville fractional derivative by

$$
\left({ }^{C} D_{0+}^{\alpha} u\right)(t):=\left(D_{0+}^{\alpha}\left[u(s)-\sum_{k=0}^{n-1} \frac{u^{(k)}(0)}{k !} s^{k}\right]\right)(t),
$$

where

$$
n= \begin{cases}{[\alpha]+1,} & \alpha \notin \mathbb{N} \\ \alpha, & \alpha \in \mathbb{N} .\end{cases}
$$

Lemma 5. Let $n$ be given by (7) and $u \in A C^{n}[0,1]$ or $u \in$ $C^{n}[0,1]$. Then

$$
\left(I_{0+}^{\alpha{ }^{C}} D_{0+}^{\alpha} u\right)(t)=u(t)+c_{0}+c_{1} t+c_{2} t^{2}+\cdots+c_{n-1} t^{n-1},
$$

where $c_{i} \in \mathbb{R}, i=0,1, \ldots, n-1$.

\section{Main Results}

In the remainder of this paper, we always assume that $\alpha \epsilon$ $(2,3]$ is a real number and $f:[0,1] \times[0,+\infty) \rightarrow[0,+\infty)$ is continuous.

Lemma 6. Let $y \in C[0,1]$ be a given function. Then the BVP

$$
\begin{aligned}
\left({ }^{C} D_{0+}^{\alpha} u\right)(t) & =y(t), \quad t \in[0,1], \\
u(0) & =0, \\
u^{\prime}(0)+u^{\prime \prime}(0) & =0, \\
u^{\prime}(1)+u^{\prime \prime}(1) & =0
\end{aligned}
$$

has a unique solution:

$$
u(t)=\int_{0}^{1} G(t, s) y(s) d s, \quad t \in[0,1],
$$

where

$$
G(t, s)= \begin{cases}\frac{(t-s)^{\alpha-1}}{\Gamma(\alpha)}+\frac{\left(t-t^{2} / 2\right)(1-s)^{\alpha-2}}{\Gamma(\alpha-1)}+\frac{\left(t-t^{2} / 2\right)(1-s)^{\alpha-3}}{\Gamma(\alpha-2)}, & 0 \leq s<t \leq 1, \\ \frac{\left(t-t^{2} / 2\right)(1-s)^{\alpha-2}}{\Gamma(\alpha-1)}+\frac{\left(t-t^{2} / 2\right)(1-s)^{\alpha-3}}{\Gamma(\alpha-2)}, & 0 \leq t \leq s<1 .\end{cases}
$$

Proof. In view of the equation in (9) and Lemma 5, we know that

$$
\begin{aligned}
u(t) & =\left(I_{0+}^{\alpha} y\right)(t)-c_{0}-c_{1} t-c_{2} t^{2} \\
& =\frac{1}{\Gamma(\alpha)} \int_{0}^{t}(t-s)^{\alpha-1} y(s) d s-c_{0}-c_{1} t-c_{2} t^{2},
\end{aligned}
$$

and so,

$$
\begin{aligned}
& u^{\prime}(t)=\frac{1}{\Gamma(\alpha-1)} \int_{0}^{t}(t-s)^{\alpha-2} y(s) d s-c_{1}-2 c_{2} t, \\
& u^{\prime \prime}(t)=\frac{1}{\Gamma(\alpha-2)} \int_{0}^{t}(t-s)^{\alpha-3} y(s) d s-2 c_{2},
\end{aligned}
$$


which together with the boundary conditions in (9) implies that

$$
\begin{aligned}
& c_{0}=0, \\
& c_{1}=-\left[\left(I_{0+}^{\alpha-1} y\right)(1)+\left(I_{0+}^{\alpha-2} y\right)(1)\right], \\
& c_{2}=\frac{1}{2}\left[\left(I_{0+}^{\alpha-1} y\right)(1)+\left(I_{0+}^{\alpha-2} y\right)(1)\right] .
\end{aligned}
$$

Therefore, BVP (9) has a unique solution:

$$
\begin{aligned}
& u(t)=\frac{1}{\Gamma(\alpha)} \int_{0}^{t}(t-s)^{\alpha-1} y(s) d s+\frac{t}{\Gamma(\alpha-1)} \int_{0}^{1}(1 \\
& -s)^{\alpha-2} y(s) d s+\frac{t}{\Gamma(\alpha-2)} \int_{0}^{1}(1-s)^{\alpha-3} y(s) d s \\
& -\frac{t^{2}}{2 \Gamma(\alpha-1)} \int_{0}^{1}(1-s)^{\alpha-2} y(s) d s-\frac{t^{2}}{2 \Gamma(\alpha-2)} \\
& \cdot \int_{0}^{1}(1-s)^{\alpha-3} y(s) d s=\int_{0}^{t}\left[\frac{(t-s)^{\alpha-1}}{\Gamma(\alpha)}\right. \\
& \left.+\frac{\left(t-t^{2} / 2\right)(1-s)^{\alpha-2}}{\Gamma(\alpha-1)}+\frac{\left(t-t^{2} / 2\right)(1-s)^{\alpha-3}}{\Gamma(\alpha-2)}\right] \\
& \cdot y(s) d s+\int_{t}^{1}\left[\frac{\left(t-t^{2} / 2\right)(1-s)^{\alpha-2}}{\Gamma(\alpha-1)}\right. \\
& \left.+\frac{\left(t-t^{2} / 2\right)(1-s)^{\alpha-3}}{\Gamma(\alpha-2)}\right] y(s) d s=\int_{0}^{1} G(t, s) \\
& \cdot y(s) d s, \quad t \in[0,1] .
\end{aligned}
$$

Now, for $s \in[0,1)$, we denote

$$
\begin{aligned}
& M(s)=\frac{(1-s)^{\alpha-1}}{\Gamma(\alpha)}+\frac{(1-s)^{\alpha-2}}{2 \Gamma(\alpha-1)}+\frac{(1-s)^{\alpha-3}}{2 \Gamma(\alpha-2)}, \\
& \gamma(s) \\
& \quad=\frac{5(\alpha-1)(1-s)+5(\alpha-1)(\alpha-2)}{18(1-s)^{2}+9(\alpha-1)(1-s)+9(\alpha-1)(\alpha-2)} .
\end{aligned}
$$

Remark 7. From the definition of function $\gamma$, it is not difficult to get that $\gamma(s) \geq \gamma(0)$ for $s \in[0,1)$ and $\gamma(0) \in(0,1)$.

Lemma 8. Green's function $G(t, s)$ defined by (11) has the following properties. Consider

(1) $G(t, s) \in C([0,1] \times[0,1))$ and $G(t, s)>0$ for $(t, s) \in$ $(0,1] \times[0,1)$

(2)

$$
\begin{aligned}
& G(t, s) \leq M(s), \quad(t, s) \in[0,1] \times[0,1), \\
& G(t, s) \geq \gamma(s) M(s), \quad(t, s) \in\left[\frac{1}{3}, \frac{2}{3}\right] \times[0,1) .
\end{aligned}
$$

Proof. Since (1) is obvious, we only need to prove (2). First, for any $(t, s) \in[0,1] \times[0,1)$, if $s<t$, then

$$
\begin{aligned}
G(t, s)= & \frac{(t-s)^{\alpha-1}}{\Gamma(\alpha)}+\frac{\left(t-t^{2} / 2\right)(1-s)^{\alpha-2}}{\Gamma(\alpha-1)} \\
& +\frac{\left(t-t^{2} / 2\right)(1-s)^{\alpha-3}}{\Gamma(\alpha-2)} \\
\leq & \frac{(1-s)^{\alpha-1}}{\Gamma(\alpha)}+\frac{(1-s)^{\alpha-2}}{2 \Gamma(\alpha-1)}+\frac{(1-s)^{\alpha-3}}{2 \Gamma(\alpha-2)} \\
= & M(s),
\end{aligned}
$$

and if $t \leq s$, then

$$
\begin{aligned}
G(t, s)= & \frac{\left(t-t^{2} / 2\right)(1-s)^{\alpha-2}}{\Gamma(\alpha-1)}+\frac{\left(t-t^{2} / 2\right)(1-s)^{\alpha-3}}{\Gamma(\alpha-2)} \\
\leq & \frac{\left(s-s^{2} / 2\right)(1-s)^{\alpha-2}}{\Gamma(\alpha-1)} \\
& +\frac{\left(s-s^{2} / 2\right)(1-s)^{\alpha-3}}{\Gamma(\alpha-2)} \\
< & \frac{(1-s)^{\alpha-2}}{2 \Gamma(\alpha-1)}+\frac{(1-s)^{\alpha-3}}{2 \Gamma(\alpha-2)}<M(s) .
\end{aligned}
$$

Next, for any $(t, s) \in[1 / 3,2 / 3] \times[0,1)$, if $s<t$, then

$$
\begin{aligned}
G(t, s)= & \frac{(t-s)^{\alpha-1}}{\Gamma(\alpha)}+\frac{\left(t-t^{2} / 2\right)(1-s)^{\alpha-2}}{\Gamma(\alpha-1)} \\
& +\frac{\left(t-t^{2} / 2\right)(1-s)^{\alpha-3}}{\Gamma(\alpha-2)} \\
> & \frac{\left(t-t^{2} / 2\right)(1-s)^{\alpha-2}}{\Gamma(\alpha-1)} \\
& +\frac{\left(t-t^{2} / 2\right)(1-s)^{\alpha-3}}{\Gamma(\alpha-2)} \\
\geq & \frac{5}{18}\left[\frac{(1-s)^{\alpha-2}}{\Gamma(\alpha-1)}+\frac{(1-s)^{\alpha-3}}{\Gamma(\alpha-2)}\right] \\
= & \gamma(s) M(s),
\end{aligned}
$$

and if $t \leq s$, then

$$
\begin{aligned}
G(t, s) & =\frac{\left(t-t^{2} / 2\right)(1-s)^{\alpha-2}}{\Gamma(\alpha-1)}+\frac{\left(t-t^{2} / 2\right)(1-s)^{\alpha-3}}{\Gamma(\alpha-2)} \\
& \geq \frac{5}{18}\left[\frac{(1-s)^{\alpha-2}}{\Gamma(\alpha-1)}+\frac{(1-s)^{\alpha-3}}{\Gamma(\alpha-2)}\right]=\gamma(s) M(s) .
\end{aligned}
$$


Let $E=C[0,1]$ be equipped with norm $\|u\|=$ $\max _{t \in[0,1]}|u(t)|$ and

$$
\begin{aligned}
K & =\left\{u \in E: u(t) \geq 0, t \in[0,1], \min _{t \in[1 / 3,2 / 3]} u(t)\right. \\
& \geq \gamma(0)\|u\|\} .
\end{aligned}
$$

Then it is easy to check that $E$ is a Banach space and $K$ is a cone in $E$.

Now, we define an operator $T$ on $K$ by

$$
(T u)(t)=\int_{0}^{1} G(t, s) f(s, u(s)) d s,
$$

$$
u \in K, t \in[0,1] \text {. }
$$

Since $G(t, s)$ is singular at $s=1$, we need to prove that operator $T$ is well defined. In fact, for any fixed $u \in K$, we have $0 \leq u(s) \leq\|u\|, s \in[0,1]$. If we let

$$
L=\max _{(s, x) \in[0,1] \times[0,\|u\|]} f(s, x),
$$

then

$$
f(s, u(s)) \leq L, \quad s \in[0,1],
$$

which together with Lemma 8 implies that

$$
G(t, s) f(s, u(s)) \leq L M(s), \quad(t, s) \in[0,1] \times[0,1) .
$$

Since $\int_{0}^{1} M(s) d s$ is convergent, we obtain that $\int_{0}^{1} G(t, s) f(s$, $u(s)) d s$ is uniformly convergent on $[0,1]$. This shows that operator $T$ is well defined. Furthermore, we know that if $u \in K$, then $T u \in E$ and if $u$ is a fixed point of $T$ in $K$, then $u$ is a nonnegative solution of BVP (3).

Lemma 9. $T: K \rightarrow K$ is completely continuous.

Proof. First, for any $u \in K$, in view of Lemma 8, we have

$$
\begin{aligned}
0 & \leq(T u)(t)=\int_{0}^{1} G(t, s) f(s, u(s)) d s \\
& \leq \int_{0}^{1} M(s) f(s, u(s)) d s, \quad t \in[0,1] .
\end{aligned}
$$

So,

$$
\|T u\| \leq \int_{0}^{1} M(s) f(s, u(s)) d s,
$$

which together with Lemma 8 implies that

$$
\begin{array}{rl}
(T u)(t) & =\int_{0}^{1} G(t, s) f(s, u(s)) d s \\
& \geq \int_{0}^{1} \gamma(s) M(s) f(s, u(s)) d s \\
& \geq \gamma(0) \int_{0}^{1} M(s) f(s, u(s)) d s \geq \gamma(0)\|T u\|, \\
t & t \in\left[\frac{1}{3}, \frac{2}{3}\right],
\end{array}
$$

which shows that

$$
\min _{t \in[1 / 3,2 / 3]}(T u)(t) \geq \gamma(0)\|T u\|
$$

Therefore, $T u \in K$.

Next, we prove that $T$ is continuous. Suppose that $u_{n}$ ( $n=$ $1,2, \ldots), u_{0} \in K$ and $\left\|u_{n}-u_{0}\right\| \rightarrow 0(n \rightarrow \infty)$. Then there exists $D^{*}>0$ such that for any $n, 0 \leq u_{n}(s) \leq D^{*}, s \in[0,1]$. If we let

$$
D=\max _{(s, x) \in[0,1] \times\left[0, D^{*}\right]} f(s, x),
$$

then for any $n$ and $t \in[0,1]$, in view of Lemma 8 , we have

$$
G(t, s) f\left(s, u_{n}(s)\right) \leq D M(s), \quad s \in[0,1) .
$$

By applying Lebesgue dominated convergence theorem, we get

$$
\begin{aligned}
\lim _{n \rightarrow \infty}\left(T u_{n}\right)(t) & =\lim _{n \rightarrow \infty} \int_{0}^{1} G(t, s) f\left(s, u_{n}(s)\right) d s \\
& =\int_{0}^{1} G(t, s) f\left(s, u_{0}(s)\right) d s \\
& =\left(T u_{0}\right)(t), \quad t \in[0,1],
\end{aligned}
$$

which indicates that $T$ is continuous.

Finally, we show that $T$ is compact. Assume that $P \subset K$ is a bounded set. Then there exists a constant $H^{*}>0$ such that $0 \leq u(s) \leq H^{*}, s \in[0,1]$ for any $u \in P$. In what follows, we will prove that $T(P)$ is relatively compact. Let

$$
H=\max _{(s, x) \in[0,1] \times\left[0, H^{*}\right]} f(s, x) .
$$

On the one hand, for any $y \in T(P)$, there exists $u \in P$ such that $y=T u$, and so, it follows from (34) and Lemma 8 that

$$
\begin{aligned}
0 & \leq y(t)=(T u)(t)=\int_{0}^{1} G(t, s) f(s, u(s)) d s \\
& \leq H \int_{0}^{1} M(s) d s, \quad t \in[0,1],
\end{aligned}
$$

which together with the fact that $\int_{0}^{1} M(s) d s$ is convergent implies that $T(P)$ is uniformly bounded. On the other hand, for any $\varepsilon>0$, since $\int_{0}^{1} M(s) d s$ is convergent, we may choose $\xi \in(0,1)$ such that

$$
\int_{\xi}^{1} M(s) d s<\frac{\varepsilon}{4 H}
$$

since $G(t, s)$ is uniformly continuous on $[0,1] \times[0, \xi]$, there exists $\delta>0$ such that for any $t_{1}, t_{2} \in[0,1]$ with $\left|t_{1}-t_{2}\right|<\delta$,

$$
\left|G\left(t_{1}, s\right)-G\left(t_{2}, s\right)\right|<\frac{\varepsilon}{2 H \xi}, \quad s \in[0, \xi]
$$


For any $y \in T(P)$, there exists $u \in P$ such that $y=T u$, and so, for any $t_{1}, t_{2} \in[0,1]$ with $\left|t_{1}-t_{2}\right|<\delta$, it follows from (36), (37), and Lemma 8 that

$$
\begin{aligned}
\mid y & \left(t_{1}\right)-y\left(t_{2}\right)|=|(T u)\left(t_{1}\right)-(T u)\left(t_{2}\right) \mid \\
& =\left|\int_{0}^{1}\left[G\left(t_{1}, s\right)-G\left(t_{2}, s\right)\right] f(s, u(s)) d s\right| \\
& \leq \int_{0}^{1}\left|G\left(t_{1}, s\right)-G\left(t_{2}, s\right)\right| f(s, u(s)) d s \\
& \leq H \int_{0}^{1}\left|G\left(t_{1}, s\right)-G\left(t_{2}, s\right)\right| d s \\
& =H\left[\int_{0}^{\xi}\left|G\left(t_{1}, s\right)-G\left(t_{2}, s\right)\right| d s\right. \\
& \left.+\int_{\xi}^{1}\left|G\left(t_{1}, s\right)-G\left(t_{2}, s\right)\right| d s\right] \\
& \leq H\left[\int_{0}^{\xi}\left|G\left(t_{1}, s\right)-G\left(t_{2}, s\right)\right| d s+2 \int_{\xi}^{1} M(s) d s\right] \\
& <H\left(\frac{\varepsilon}{2 H}+\frac{\varepsilon}{2 H}\right)=\varepsilon,
\end{aligned}
$$

which indicates that $T(P)$ is equicontinuous. By Arzela-Ascoli theorem, we know that $T(P)$ is relatively compact. Therefore, $T: K \rightarrow K$ is completely continuous.

For convenience, we denote

$$
\begin{aligned}
& A=\left[\int_{0}^{1} M(s) d s\right]^{-1}, \\
& B=\left[\int_{1 / 3}^{2 / 3} \gamma(s) M(s) d s\right]^{-1} .
\end{aligned}
$$

Theorem 10. Assume that there exist two different positive constants $r_{1}$ and $r_{2}$ such that the following conditions are fulfilled:

$$
\begin{aligned}
& f(t, x) \leq A r_{1}, \quad(t, x) \in[0,1] \times\left[0, r_{1}\right], \\
& f(t, x) \geq B r_{2}, \quad(t, x) \in\left[\frac{1}{3}, \frac{2}{3}\right] \times\left[\gamma(0) r_{2}, r_{2}\right] .
\end{aligned}
$$

Then BVP (3) has a positive solution $u$ with $\min \left\{r_{1}, r_{2}\right\} \leq\|u\| \leq$ $\max \left\{r_{1}, r_{2}\right\}$.

Proof. Without loss of generality, we assume that $r_{1}<r_{2}$. Let

$$
\begin{aligned}
& \Omega_{1}=\left\{u \in E:\|u\|<r_{1}\right\}, \\
& \Omega_{2}=\left\{u \in E:\|u\|<r_{2}\right\} .
\end{aligned}
$$

Then for any $u \in K \cap \partial \Omega_{1}$, we get $0 \leq u(s) \leq r_{1}, s \in[0,1]$, which together with (40) and Lemma 8 implies that

$$
\begin{aligned}
0 & \leq(T u)(t)=\int_{0}^{1} G(t, s) f(s, u(s)) d s \\
& \leq A r_{1} \int_{0}^{1} M(s) d s=r_{1}=\|u\|, \quad t \in[0,1] .
\end{aligned}
$$

This shows that

$$
\|T u\| \leq\|u\| \quad \text { for } u \in K \cap \partial \Omega_{1} .
$$

For any $u \in K \cap \partial \Omega_{2}$, we get $\gamma(0) r_{2} \leq u(s) \leq r_{2}, s \in[1 / 3,2 / 3]$, which together with (41) and Lemma 8 implies that

$$
\begin{aligned}
(T u)\left(\frac{1}{2}\right) & =\int_{0}^{1} G\left(\frac{1}{2}, s\right) f(s, u(s)) d s \\
& \geq \int_{1 / 3}^{2 / 3} G\left(\frac{1}{2}, s\right) f(s, u(s)) d s \\
& \geq B r_{2} \int_{1 / 3}^{2 / 3} \gamma(s) M(s) d s=r_{2}=\|u\| .
\end{aligned}
$$

This indicates that

$$
\|T u\| \geq\|u\| \quad \text { for } u \in K \cap \partial \Omega_{2} .
$$

Therefore, it follows from Theorem 1, Lemma 9, (44), and (46) that the operator $T$ has a fixed point $u \in K \cap\left(\bar{\Omega}_{2} \backslash \Omega_{1}\right)$, which is a desired positive solution of BVP (3).

Example 11. We consider the BVP

$$
\begin{aligned}
\left({ }^{C} D_{0+}^{5 / 2} u\right)(t) & =\frac{867 \sqrt{\pi} t u^{2}(t)}{25}, \quad t \in[0,1], \\
u(0) & =0, \\
u^{\prime}(0)+u^{\prime \prime}(0) & =0, \\
u^{\prime}(1)+u^{\prime \prime}(1) & =0 .
\end{aligned}
$$

Since $\alpha=5 / 2$, a direct calculation shows that $M(s)$

$$
\begin{aligned}
= & \frac{1}{\sqrt{\pi}}\left[\frac{4}{3}(1-s)^{3 / 2}+(1-s)^{1 / 2}+\frac{1}{2}(1-s)^{-1 / 2}\right], \\
\gamma(s) & =\frac{5}{3}\left[\frac{2(1-s)+1}{8(1-s)^{2}+6(1-s)+3}\right] .
\end{aligned}
$$

So,

$$
\begin{aligned}
\gamma(0) & =\frac{5}{17}, \\
A & =\frac{5 \sqrt{\pi}}{11}, \\
B & =\frac{81 \sqrt{3 \pi}(13 \sqrt{2}+11)}{1085} .
\end{aligned}
$$

Let $f(t, x)=867 \sqrt{\pi} t x^{2} / 25,(t, x) \in[0,1] \times[0,+\infty)$. If we choose $r_{1}=125 / 9537$ and $r_{2}=81 \sqrt{3}(13 \sqrt{2}+11) / 1085$, then it is easy to verify that

$$
\begin{aligned}
& f(t, x) \leq f\left(1, \frac{125}{9537}\right)=A r_{1}, \quad(t, x) \in[0,1] \times\left[0, \frac{125}{9537}\right], \\
& f(t, x) \geq f\left(\frac{1}{3}, \frac{81 \sqrt{3}(13 \sqrt{2}+11)}{3689}\right)=B r_{2}, \\
& (t, x) \in\left[\frac{1}{3}, \frac{2}{3}\right] \times\left[\frac{81 \sqrt{3}(13 \sqrt{2}+11)}{3689}, \frac{81 \sqrt{3}(13 \sqrt{2}+11)}{1085}\right] .
\end{aligned}
$$


So, all the hypotheses of Theorem 10 are fulfilled. Therefore, it follows from Theorem 10 that BVP (47) has a positive solution $u$ satisfying

$$
\frac{125}{9537} \leq\|u\| \leq \frac{81 \sqrt{3}(13 \sqrt{2}+11)}{1085} .
$$

\section{Conflict of Interests}

The authors declare that there is no conflict of interests regarding the publication of this paper.

\section{References}

[1] A. A. Kilbas, H. M. Srivastava, and J. J. Trujillo, Theory and Applications of Fractional Differential Equations, Elsevier, Amsterdam, The Netherlands, 2006.

[2] W. Feng, S. Sun, X. Li, and M. Xu, "Positive solutions to fractional boundary value problems with nonlinear boundary conditions," Boundary Value Problems, vol. 2014, article 225, 2014.

[3] D. Q. Jiang and C. J. Yuan, "The positive properties of the Green function for Dirichlet-type boundary value problems of nonlinear fractional differential equations and its application," Nonlinear Analysis, Theory, Methods and Applications, vol. 72, no. 2, pp. 710-719, 2010.

[4] Z.-W. Lv, "Positive solutions of m-point boundary value problems for fractional differential equations," Advances in Difference Equations, vol. 2011, Article ID 571804, 2011.

[5] W. G. Yang, "Positive solutions for nonlinear Caputo fractional differential equations with integral boundary conditions," Journal of Applied Mathematics and Computing, vol. 44, no. 1-2, pp. 39-59, 2014.

[6] S. Q. Zhang, "Positive solutions for boundary-value problems of nonlinear fractional differential equations," Electronic Journal of Differential Equations, vol. 2006, article 36, 12 pages, 2006.

[7] Y. D. Ji, Y. P. Guo, J. Q. Qiu, and L. Y. Yang, "Existence of positive solutions for a boundary value problem of nonlinear fractional differential equations," Advances in Difference Equations, vol. 2015, article 13, 2015.

[8] C. F. Li, X. N. Luo, and Y. Zhou, "Existence of positive solutions of the boundary value problem for nonlinear fractional differential equations," Computers and Mathematics with Applications, vol. 59, no. 3, pp. 1363-1375, 2010.

[9] W. Z. Xie, J. Xiao, and Z. G. Luo, "Existence of extremal solutions for nonlinear fractional differential equation with nonlinear boundary conditions," Applied Mathematics Letters, vol. 41, pp. 46-51, 2015.

[10] J. F. Xu, Z. L. Wei, and W. Dong, "Uniqueness of positive solutions for a class of fractional boundary value problems," Applied Mathematics Letters, vol. 25, no. 3, pp. 590-593, 2012.

[11] K. Zhao and P. Gong, "Existence of positive solutions for a class of higher-order Caputo fractional differential equation," Qualitative Theory of Dynamical Systems, vol. 14, no. 1, pp. 157171, 2015.

[12] Z. B. Bai and T. T. Qiu, "Existence of positive solution for singular fractional differential equation," Applied Mathematics and Computation, vol. 215, no. 7, pp. 2761-2767, 2009.

[13] X. Q. Zhang, L. Wang, and Q. Sun, "Existence of positive solutions for a class of nonlinear fractional differential equations with integral boundary conditions and a parameter," Applied Mathematics and Computation, vol. 226, pp. 708-718, 2014.

[14] D. J. Guo and V. Lakshmikantham, Nonlinear Problems in Abstract Cones, Academic Press, New York, NY, USA, 1988.

[15] M. A. Krasnoselskii, Positive Solutions of Operator Equations, Noordhoff, Groningen, The Netherlands, 1964. 


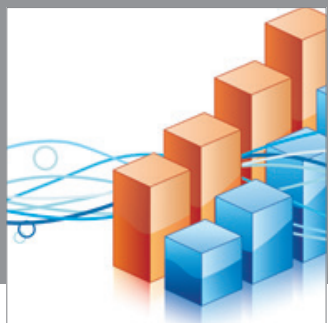

Advances in

Operations Research

mansans

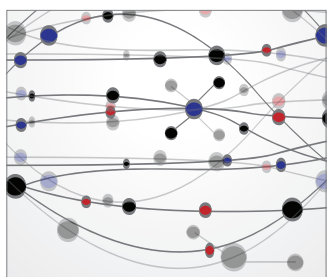

The Scientific World Journal
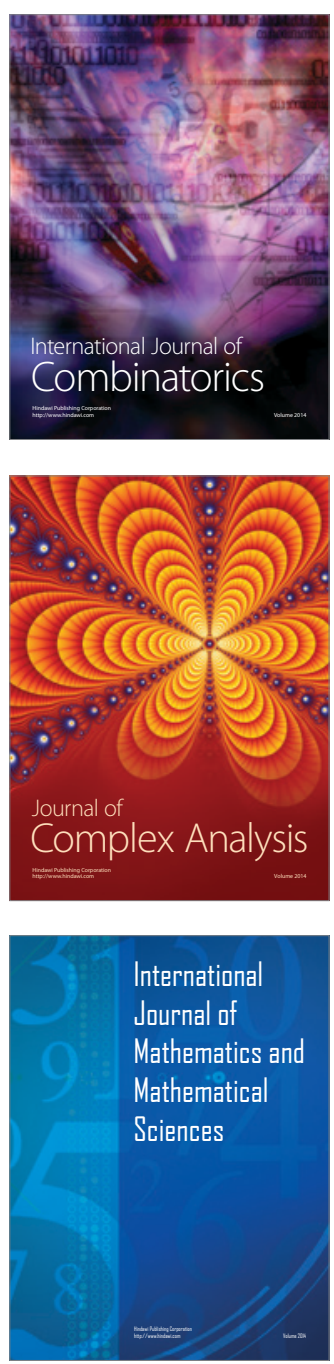
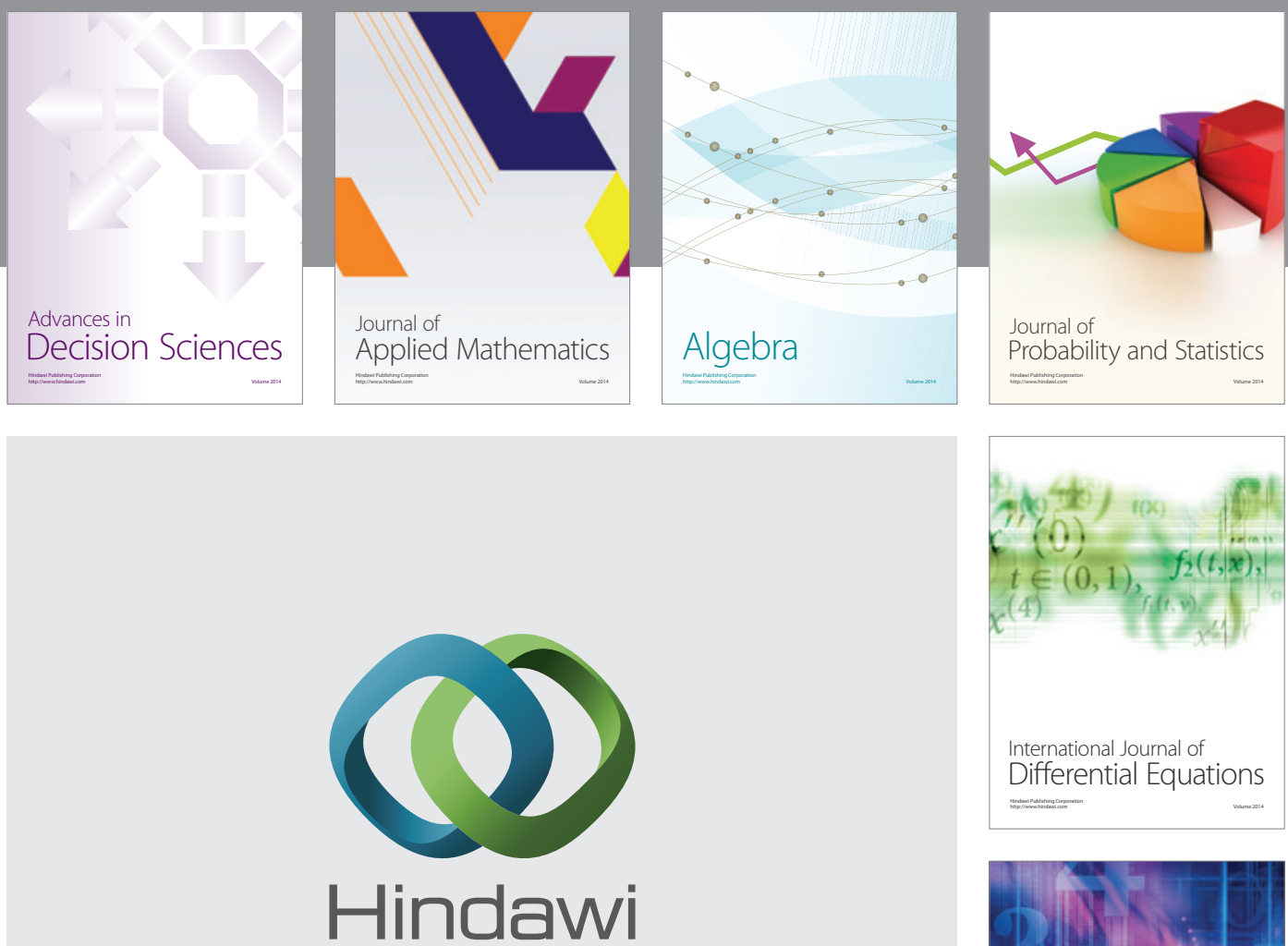

Submit your manuscripts at http://www.hindawi.com
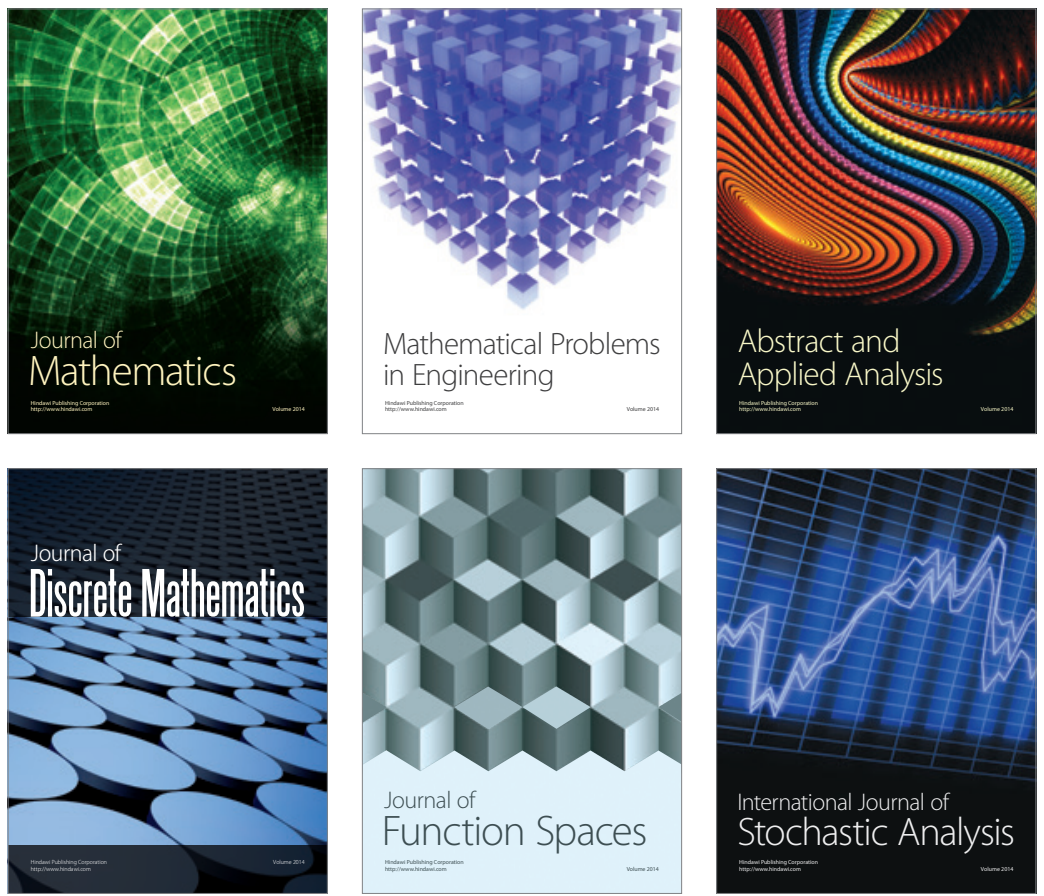

Journal of

Function Spaces

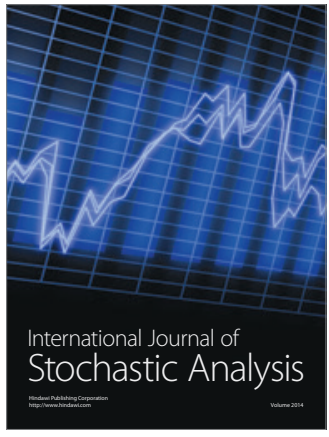

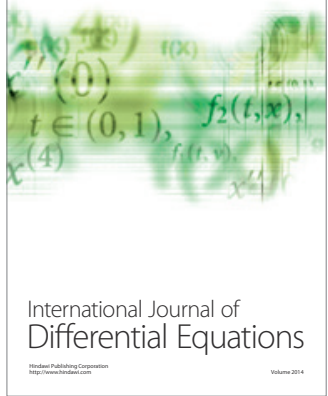
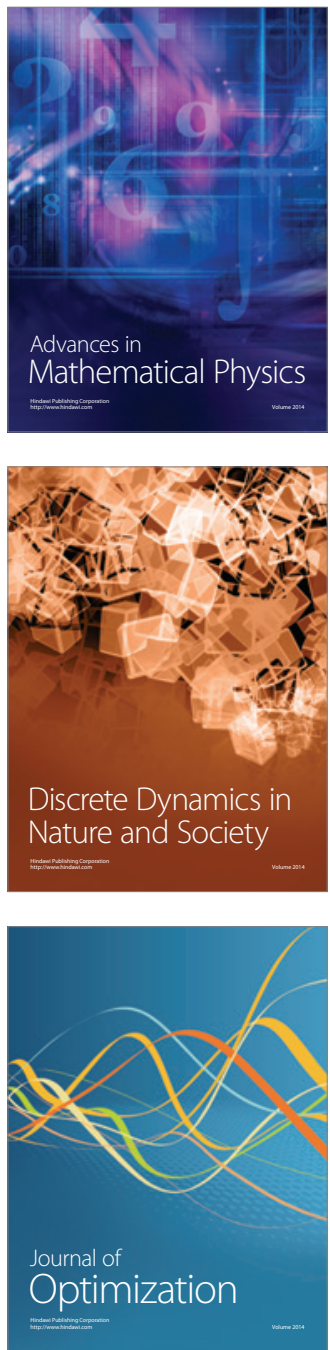